\title{
In vitro antiplasmodium and chloroquine resistance reversal effects of mangostin
}

\begin{abstract}
Aim/Background: Chloroquine (CQ) resistance that appeared among different strains of Plasmodium falciparum is considered as the worst catastrophe in the realm of malaria chemotherapy. CQ is still the most favorable drug among other antimalarials especially in the poor endemic areas due to its high potency and cost-effectiveness. This urged the scientists to explore for other alternatives or sensitizers for CQ. Materials and Methods: In this experiment, the antiplasmodium and the CQ resistance reversing effects of mangostin were tested using the in vitro SYBRE green-1-based drug sensitivity assay and the isobologram technique, respectively. Furthermore, its safety level toward two types of mammalian cells, namely Vero cells and red blood cells (RBCs), was screened using the 3-(4,5-dimethylthiazol-2-yl)-2,5diphenyltetrazolium bromide-based drug sensitivity and the RBCs hemolysis assays, respectively. On the other hand, its effect against hemozoin formation was screened using $\beta$ hematin formation. Meanwhile, its molecular characters were determined the in silico on-line free chemi-informatic Molinspiration software for the molecular characterization as well as the standard testes for the measurement of the antioxidant effect. Results: Mangostin was moderately effective and selective toward the plasmodium so it is unsuitable to be a substituent for CQ. But it improved the sensitivity of the parasite to CQ. The molecular elucidation suggests that its CQ resistance reversal effect can be ascribed to its ability to interfere with hemozoin formation or the intravacuolar accumulation of CQ. Conclusion: Overall, the study suggests mangostin as a possible pharmacophore to develop new CQ resistance reversing agents but further studies are recommended to confirm this notion.
\end{abstract}

Keyword: Chloroquine; Falciparum and SYBR green-I; Isobologram; Mangostine; Resistance 